\title{
QCD in magnetic field, Landau levels and double-life of unbroken center-symmetry
}

\author{
Mohamed M. Anber ${ }^{a}$ and Mithat Ünsal ${ }^{b}$ \\ ${ }^{a}$ Department of Physics, University of Toronto, \\ Toronto, ON M5S 1A\%, Canada \\ ${ }^{b}$ Department of Physics and Astronomy, SFSU, \\ San Francisco, CA 94132, U.S.A. \\ E-mail: mohamed.anber@epfl.ch, unsal.mithat@gmail.com
}

ABSTRACT: We study the thermal confinement/deconfinement and non-thermal quantum phase transitions or rapid cross-overs in QCD and QCD-like theories in external magnetic fields. At large magnetic fields, while the contribution of gauge fluctuations to Wilson-line potential remains unaltered at one-loop order, the contribution of fermions effectively becomes two lower dimensional and is enhanced by the density of states of the lowest Landau level (LLL). In a spatial compactification and for heavy adjoint fermions, this enhancement leads to a calculable zero temperature quantum phase transition on $\mathbb{R}^{3} \times \mathbb{S}^{1}$ driven by a competition between the center-destabilizing gauge contribution and center-stabilizing LLL fermions. We also show that at a (formal) asymptotically large magnetic field, the adjoint fermions with arbitrarily large but fixed mass stabilize the center symmetry. This is an exotic case of simultaneous non-decoupling of large mass fermions (due to the enhancement by the LLL density of states) and decoupling from the low energy effective field theory. This observation has important implications for both Hosotani mechanism, for which gauge symmetry "breaking" occurs, and large- $N$ volume independence (Eguchi-Kawai reduction), for which gauge structure is never "broken". Despite sounding almost self-contradictory, we carefully explain the physical scales entering the problem, double-meaning of unbroken center symmetry and how a clash is avoided. We also identify, for both thermal and spatial compactification, the jump in magnetic susceptibility as an order parameter for the deconfinement transition. The predictions of our analysis are testable by using current lattice techniques.

Keywords: Confinement, Field Theories in Lower Dimensions, Phase Diagram of QCD, Nonperturbative Effects

ARXIV EPRINT: 1309.4394 


\section{Contents}

1 Introduction 1

2 Turning on magnetic field in QCD on $\mathbb{R}^{3} \times \mathbb{S}^{1} \quad 4$

$\begin{array}{lll}3 & \text { Landau levels and the role of the lowest Landau level } & 7\end{array}$

3.1 Magnetic susceptibility and its jump across the deconfinement transition $\quad 9$

4 Massive QCD(adj) in external magnetic field 11

$\begin{array}{lll}4.1 & \text { Abelian confinement and large- } N \text { volume independence regimes } & 12\end{array}$

5 Comments on lattice realization of abelian confinement and Hosotani mechanism on $\mathbb{R}^{3} \times S^{1} \quad 14$

5.1 The resolution of Eguchi-Kawai versus Hosotani puzzle 16

5.2 How large should the box be in order to see the setting of mass gap and abelian confinement?

5.3 Why is the weak coupling corner important both for lattice and continuum studies?

\section{Introduction}

Quarks carry both non-abelian color and abelian electric charges. In relativistic heavy ion collisions (RHIC), large external $\mathrm{U}(1)_{\mathrm{em}}$ magnetic field (of order $\sqrt{|e B|} \sim 10^{2} \mathrm{MeV}$ ) is generated. This is parametrically of order QCD-strong scale. Therefore, it is of experimental interest to study both equilibrium thermodynamics and non-equilibrium properties of QCD in external $B$-fields.

A magnetic field introduces a Landau level structure to the fermion spectrum. Few rather interesting phenomena stem from this: chiral magnetic effect which is an interplay of the LLL structure and topological aspects of QCD [1-5] and magnetic catalysis which helps spontaneous breaking of non-abelian chiral symmetry even at very weak coupling [6-10], inverse magnetic catalysis and non-monotoniticity observed in lattice simulations [11-14]. Also see $[15,16]$ for simulations of QCD in external $B$-field.

In this work, our goal is to study the the role of the $B$-fields in center-symmetry realization, and the equilibrium thermodynamics and some aspects of phase structure for QCD-like theories, with fermions in one and two-index representations $\mathcal{R}$. An interesting question is whether the back-reaction of the fermions in varying- $B$ field can alter the phase of the theory, say, from a center-broken phase to a center symmetric phase or vice versa. We find an example of such phenomena for adjoint representation fermions. Another interesting 
question is the interplay of external- $\mathrm{U}(1)_{\mathrm{em}} B$-fields, monopole-instantons (with fractional topological charge) which carry chromomagnetic $B$-field, and chiral symmetry realization which we study in a follow-up.

We study center-symmetry realization in both thermal and spatial compactification in the presence of external magnetic fields. In path integral formalism, integrating out fermions with anti-periodic (periodic) spin connection correspond to the thermal (twisted) partition function. In operator formalism, this amounts to regular (graded) trace over the Hilbert space, namely

$$
\begin{aligned}
Z_{\eta} & =Z_{\mathcal{B}}-\eta Z_{\mathcal{F}}=\operatorname{tr}\left(e^{-L H}(-\eta)^{F}\right) \\
& =\int_{A_{\mu}(L)=A_{\mu}(0)} D A_{\mu} e^{-S[A]} \int_{\psi(L)=\eta \psi(0)} D \psi D \bar{\psi} e^{\int_{\mathbb{R}^{3} \times \mathbb{S}_{\eta}^{1}} \bar{\psi}(-i \not D+m) \psi} \\
& =\int_{A_{\mu}(L)=A_{\mu}(0)} D A_{\mu} e^{-S[A]} \operatorname{det}_{\eta}(-i \not D+m), \\
\eta & =\left\{\begin{array}{lll}
- & \text { thermal circle, } \mathbb{S}_{-}^{1} \\
+ & \text { spatial (non }- \text { thermal) circle, } \mathbb{S}_{+}^{1} & L=\beta=1 / T,
\end{array}\right.
\end{aligned}
$$

where $(-1)^{F}$ is fermion number modulo two, acting as \pm on bosonic (fermionic) Hilbert spaces, and $\operatorname{det}_{\mp}$ corresponds to the determinant in the space of anti-periodic/periodic functions. ${ }^{1}$ By studying the properties of the Dirac operator $(-i \not D+m)$ in the presence of external magnetic field and a background Wilson line, we find the fermion induced oneloop potential. ${ }^{2}$ Calculationally, this is a standard generalization of the Euler-Heisenberg effective Lagrangian (see e.g. [17, 18] and references therein) and Gross-Pisarski-Yaffe oneloop potential for Wilson line [19-27]. We express the fermion induced Wilson line potential as a sum over Landau level contributions.

Consider QCD with gauge group $G$ with fermions in representation $\mathcal{R}$, where fermions also carry charges under $\mathrm{U}(1)_{\mathrm{em}}$. For $\mathcal{R}$, we primarily consider $n_{f}$ fundamental (F), antisymmetric (AS), symmetric (S) Dirac fermions and $n_{f}$ adjoint (adj) Weyl fermions. For adjoint matter, we only consider $n_{f}=$ even so that we can build $n_{f} / 2$ Dirac fermions to which we can assign an electric charge without causing any gauge anomaly. The motivation to study two-index representation is that $\mathrm{QCD}(\mathrm{AS})$ is a natural generalization of ordinary QCD to large- $N$, and it is related via orientifold equivalence to the adjoint representation [26, 28, 29].

Thermal compactification and phase structure. At strong magnetic fields, the fermion induced Wilson line potential is dominated by the lowest Landau level (LLL), and

\footnotetext{
${ }^{1}$ It is important to note that the periodic boundary condition for fermions is not unphysical, it has a well-defined meaning in operator formalism. In either case, fermions are spin-half particles and they obey the Pauli exclusion principle, and anti-commutation relations. However, the spatial compactification does not have a thermal interpretation, and the Fermi-Dirac distribution (relevant to thermal QFT) only arise in the thermal compactification.

${ }^{2}$ We take the U(1)em magnetic field as an external field, with no dynamics associated with it. Otherwise, at small- $L$ and vanishing fermion mass, the abelian part would be strongly coupled.
} 


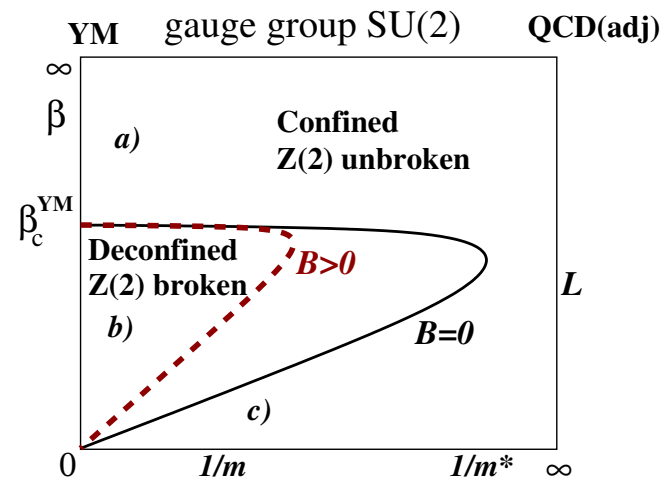

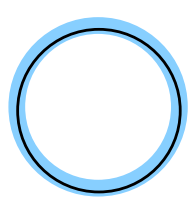

a)

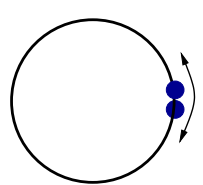

b)

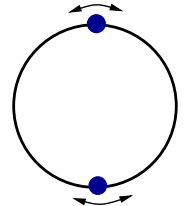

c)

Figure 1. Left: The phase diagram for $\mathrm{SU}(2)$ gauge theory with $n_{f}=2$ adjoint Weyl fermions, on $\mathbb{R}^{3} \times \mathbb{S}_{+}^{1}$, in the $L-1 / m$ plane for $B=0$ and $|B|>0$. Center-broken regime shrinks with increasing magnetic field. Right: a) "Cartoon" of strong coupling non-trivial holonomy $\langle\operatorname{tr} \Omega\rangle=0$, eigenvalues are randomized over the unit circle. b) Weak coupling trivial holonomy $\langle\operatorname{tr} \Omega\rangle=1$. c) Weak coupling non-trivial holonomy $\langle\operatorname{tr} \Omega\rangle=0$, eigenvalues are at anti-podal points, and the fluctuations in their position is small. a) and c) domains are both center-symmetric and are continuously connected.

undergoes dimensional reduction by two dimensions, similar to the chiral condensate [8-10]:

$$
\mathcal{V}_{-}[\Omega]=\mathcal{V}_{\text {gauge }}^{\mathbb{R}^{3} \times S_{\beta}^{1}}[\Omega]+\mathcal{V}_{-, \mathcal{R}}^{\mathbb{R}^{3} \times S_{\beta}^{1}}[\Omega] \quad \stackrel{\text { large-B }}{\longrightarrow} \quad \mathcal{V}_{\text {gauge }^{\mathbb{R}^{3}} \times S_{\beta}^{1}}^{1}[\Omega]+\left(\frac{e B}{2 \pi}\right) \mathcal{V}_{-, \mathcal{R}}^{\mathbb{R}^{1} \times S_{\beta}^{1}}[\Omega],
$$

where $\left(\frac{e B}{2 \pi}\right)$ is the density of states of the LLL. $\mathcal{V}_{\text {gauge }}^{\mathbb{R}^{3} \times S_{\beta}^{1}}[\Omega]$ is the standard contribution of gauge fluctuation to the Wilson line potential [19]. For fermions, life becomes essentially two dimensional. If $\vec{B}=B \hat{z}$, then, one effectively deletes the $x y$-plane and the fermions are localized to the two-dimensional $z t$ plane. Furthermore, their effect is parametrically enhanced by $\left(\frac{e B}{T^{2}}\right)=\left(\frac{\beta}{\ell_{m}}\right)^{2} \gg 1$ where $\ell_{m} \sim 1 / \sqrt{e B}$ is the magnetic length scale. Extremizing the potential yields free energy density, given by

$$
\mathcal{F}=-\operatorname{dim}(\operatorname{adj}) \times \underbrace{\left(\frac{\pi^{2}}{45} T^{4}\right)}_{\text {Stefan-Boltzmann } 4 \mathrm{~d}}-n_{f} \operatorname{dim}(\mathcal{R}) \times \underbrace{\left(\frac{|e B|}{2 \pi}\right)}_{\text {LLL density of states }} \times \underbrace{\left(\frac{\pi}{12} T^{2}\right)}_{\text {Stefan-Boltzmann } 2 \mathrm{~d}}
$$

in accordance with the LLL interpretation and dimensional reduction.

Spatial compactification and a quantum phase transition. An interesting gauge phenomenon occurs for $\mathcal{R}=\operatorname{adj}$ where fermions (with mass $m$ ) are endowed with periodic boundary conditions, $\eta=+1$ in (1.1). When the fermions are massless or sufficiently light, they induce a center-stabilizing potential, leading to gauge "symmetry breaking" or adjoint Higgsing or abelianization at one loop-order, and in fact, to all orders in perturbation theory.

This result has two mutually independent and exclusive histories. One is in the context of gauge-Higgs unification [30-32] for which gauge symmetry breaking (Hosotani mechanism) occurs and the other is in the discussion of large- $N$ volume independence $[33,34]$ (working realization of Eguchi-Kawai reduction [35]) where gauge symmetry breaking never 
occurs, and the semi-classical calculable regime where abelianization again occurs [33]. The discussion of scales, the role of the parameter $\frac{L N \Lambda}{2 \pi}$ which determines whether a centersymmetric regime exhibits adjoint Higgsing or not first appeared more recently in $[33,36]$, in distinguishing large- $L$ or large- $N$ (gauge structure unbroken) and small- $L N$ adjoint Higgsing semi-classical calculable regimes, and did not appear in earlier work. In particular, abelianization and semi-classical calculability takes place in the $\frac{L N \Lambda}{2 \pi} \lesssim 1$ domain [33], and large- $N$ volume independence in the $\frac{L N \Lambda}{2 \pi} \gg 1$ domain $[34,37]$. (Also see more recent works $[38,39]$ emphasizing the role of $L N \Lambda$ parameter, and recent reviews of large- $N$ limits $[40,41]$.) As explained in detail in section 5, the discussion of scales clarifies how a contradiction is avoided between these two different regimes. The understanding of the role of parameter $\frac{L N \Lambda}{2 \pi}$ is extremely important in finding lattice realization of these two regimes.

When the fermions are heavy, this theory has an exotic phase structure, shown in figure 1, center-symmetric at sufficiently small and sufficiently large $S^{1}$, and center-broken in between $[37,42] .{ }^{3}$ This system is interesting because it does not have a strict thermal interpretation, but it admits a non-thermal quantum phase transition. Its phase diagram in the $L-1 / m$ plane in the absence of magnetic field is studied in [37]. We study the same phase diagram in the presence of large- $B$ fields. As shown in figure 1, the center-broken regime shrinks with the application of the $B$-field. This happens when the magnetic field is sufficiently large such that it can compensate suppression due to the mass term for fermion. This is a rather exotic phase transition driven by the competition between centerdestabilizing gauge fluctuations and the increase of the LLL density of states of the adjoint fermions endowed with periodic boundary conditions. This transition can be checked by using standard lattice simulations, by adding magnetic field to the set-up of [42].

\section{$2 \quad$ Turning on magnetic field in QCD on $\mathbb{R}^{3} \times \mathbb{S}^{1}$}

We consider $\mathrm{SU}(N)$ gauge theory coupled to massive fermions on $\mathbb{R}^{3} \times \mathbb{S}^{1}$ which obey either periodic or anti-periodic boundary conditions along $\mathbb{S}^{1}$. We couple the fermions to a background $\mathrm{U}(1)_{\mathrm{em}}$ gauge field that is taken to be constant and perpendicular to the $\mathbb{S}^{1}$ circle. In the following, it will prove easier to work with Dirac fermions. Hence, the system Lagrangian reads

$$
\mathcal{L}=-\frac{1}{4 g^{2}} F_{\mu \nu}^{a} F^{a \mu \nu}+\bar{\psi}\left(\not \partial-i A^{a} T^{a}+i e A_{\mathrm{em}}+m\right) \psi,
$$

where $e$ is the electromagnetic coupling constant and $T^{a}$ are the Lie generators in the appropriate representation $\mathcal{R}$. Next, we analytic continue to the Euclidean space and integrate out the fermions to obtain the one-loop effective action $\Gamma^{\text {Dirac }}=\operatorname{Tr} \log (-i \not D+m)$, where $\not D=\not \partial-i \not A^{a} T^{a}+i e A_{\mathrm{em}}$, and Tr denotes the trace over spacetime, Dirac and color indices. Using the fact that the sign of the fermion mass is irrelevant, we get

$$
\Gamma^{\text {Dirac }}=\operatorname{Tr} \log (-i \not D+m)=\operatorname{Tr} \log (i \not D+m)=\frac{1}{2} \operatorname{Tr} \log \left(-\not D^{2}+m^{2}\right)
$$

\footnotetext{
${ }^{3}$ The lattice simulations in [42] exhibits the existence of small and large- $L$ confined phases, but do not currently show their continuity on the small mass regime $m<m^{*}$. However, there is strong theoretical reasons to believe that the theory will not have center-broken intermediate regime for $m<m^{*}$. The reason for the non-observation in [42] may be that the simulations are not run at sufficiently light fermion masses.
} 
where $\not D^{2}=D^{2}-\sigma_{\mu \nu}\left(F^{a \mu \nu} T^{a}+e F_{\mathrm{em}}^{\mu \nu}\right) / 2$, and $\sigma_{\mu \nu}=\frac{i}{2}\left[\gamma_{\mu}, \gamma_{\nu}\right]$. The effective action $\Gamma^{\text {Dirac }}$ is a divergent quantity. Therefore, we regularize it by subtracting out the free field contribution:

$$
\Gamma_{\mathrm{reg}}^{\mathrm{Dirac}}=\frac{1}{2} \operatorname{Tr} \log \frac{\left(-\not D^{2}+m^{2}\right)}{\left(-\square+m^{2}\right)},
$$

such that we have $\Gamma_{\text {reg }}^{\text {Dirac }}=0$ as we turn off both the color and electromagnetic fields.

In general, the calculation of $\Gamma_{\text {reg }}^{\text {Dirac }}$ is a formidable task. However, it turns out that this problem can have an exact solution in a few special cases. We specify our problem by turning on a constant holonomy (or Wilson line) $A_{0}^{a}$ along the $\mathbb{S}^{1}$ direction and ignoring the gauge fluctuations in all other directions. In consequence, the chromo-field strength vanishes $F^{a \mu \nu}=0$, i.e. the non-abelian gauge connection is flat. Then, using the integral representation of the $\log$ function, we obtain

$$
\begin{aligned}
\Gamma_{\text {reg }}^{\text {Dirac }} & =\frac{1}{2} \operatorname{Tr} \log \frac{-D^{2}-e \sigma \cdot F_{\mathrm{em}} / 2+m^{2}}{-\square+m^{2}} \\
& =-\frac{1}{2} \operatorname{Tr} \int_{0}^{\infty} \frac{d \tau}{\tau}\left(e^{-\tau\left(-D^{2}-e \sigma \cdot F_{\mathrm{em}} / 2+m^{2}\right)}-e^{-\tau\left(-\square+m^{2}\right)}\right),
\end{aligned}
$$

where $D^{2}=\left(\partial_{0}+A_{0}^{a} T^{a}\right)^{2}+\left(\partial_{i}+i e A_{\mathrm{em}} i\right)^{2}$. The trace over the free field part is trivial and can be performed directly by going to the momentum space. Since the electromagnetic field is assumed to be perpendicular to $\mathbb{S}^{1}$, one can break the trace into two independent parts: one along the compact dimension and the other along the infinite dimensions as follows:

$$
\begin{aligned}
\Gamma_{\text {reg }}^{\text {Dirac }}=-\frac{1}{2} \sum_{n \in \mathbb{Z}} \int_{0}^{\infty} \frac{d \tau}{\tau} e^{-m^{2} \tau} & \left\{\operatorname{tr}_{\mathcal{R}}\left[e^{-\tau\left(\omega_{n}+A_{0}^{a} T^{a}\right)^{2}}\right] \times \operatorname{tr}\left[e^{-\tau\left[\left(\partial_{i}+i e A_{\mathrm{em} i}\right)^{2}+e \sigma \cdot F_{\mathrm{em}} / 2\right]}\right]\right. \\
& \left.-4 \int \frac{d^{3} k}{(2 \pi)^{3}} e^{-\tau\left[\left(\omega_{n}^{2}+k^{2}\right)+m^{2}\right]}\right\},
\end{aligned}
$$

where $\omega_{n}$ are the Matsubara frequencies which are given by $2 \pi n / L$ and $(2 n+1) \pi / L$ for spatial and thermal compactifications, respectively. During this process of integrating out the Kaluza-Klein modes, we have assumed that these modes are weakly coupled and can be integrated out perturbatively. This assumption is justified in the small- $L$ regime by the asymptotic freedom of QCD, for sufficiently small number of fermions. (Recall that the $\mathrm{U}(1)_{\mathrm{em}}$ magnetic field is treated as a background, with no dynamics associated with it. Otherwise, at small- $L$, the abelian part would be strongly coupled.) The first $\operatorname{trace} \operatorname{tr}_{\mathcal{R}}$ is over the Lie algebra representation $\mathcal{R}$, while the second trace is over space and Dirac indices. The trace $\operatorname{tr}\left[e^{-\tau\left[\left(\partial_{i}+i e A_{\mathrm{em}}\right)^{2}+e \sigma \cdot F \mathrm{em} / 2\right]}\right]$ is a standard Euler-Heisenberg calculation which encodes information about a constant electromagnetic field in 3 dimensions. Turning on only the magnetic field, setting the electric field to zero, we have ${ }^{4}$

$$
\operatorname{tr}\left[e^{-\tau\left[\left(\partial_{i}+i e A_{\mathrm{em}}\right)^{2}+e \sigma \cdot F_{\mathrm{em}} / 2\right]}\right]=4 \frac{V_{\mathbb{R}^{3}}}{(4 \pi \tau)^{3 / 2}} \frac{e \tau B}{\tanh (e \tau B)},
$$

\footnotetext{
${ }^{4}$ In the case of spatial compactification, the magnetic field has only a single component in $\mathbb{R}^{3}$ (recall that in this case one of the dimensions in $\mathbb{R}^{3}$ is the time dimension; in $2+1$ dimensions the magnetic field has only one component). On the other hand, in the case of thermal compactification the magnetic field can have three components. However, we can always choose the magnetic field to be aligned in the $\hat{z}$-direction. Thus, (2.6) is valid for both spatial and thermal compactifications.
} 
where $V_{\mathbb{R}^{3}}$ is the three dimensional volume. Putting things together we find

$$
\begin{aligned}
\Gamma_{\text {reg }}^{\text {Dirac }}=-2 \sum_{n \in Z} \int_{0}^{\infty} \frac{d \tau}{\tau} \frac{V_{\mathbb{R}^{3}}}{(4 \pi \tau)^{3 / 2}} e^{-m^{2} \tau} & {\left[\operatorname{tr}_{\mathcal{R}}\left(e^{-\tau\left(\omega_{n}+A_{0}^{a} T^{a}\right)^{2}}\right) \times \frac{e \tau B}{\tanh (e \tau B)}\right.} \\
& \left.-e^{-\tau \omega_{n}^{2}}\right] .
\end{aligned}
$$

At this stage, we define the effective potential $\mathcal{V}$ as $\mathcal{V} \equiv-\Gamma /\left(L V_{\mathbb{R}^{3}}\right)$. Using the Poisson resummation formula

$$
\sum_{n \in Z} e^{-\tau\left(\omega_{n}+q\right)^{2}}=\frac{L}{\sqrt{4 \pi \tau}} \sum_{n \in Z} e^{-\frac{L^{2} n^{2}}{4 \tau}+i n L q},
$$

and the change of variables $\tau=L^{2} y$, we obtain the effective potential per Dirac fermion

$$
\mathcal{V}^{\mathbb{R}^{3} \times \mathbb{S}_{ \pm}^{1}}=\frac{2}{(4 \pi)^{2} L^{4}} \sum_{n=1}^{\infty} \int_{0}^{\infty} \frac{d y}{y^{3}} e^{-\frac{n^{2}}{4 y}-m^{2} L^{2} y}\left\{\frac{e B L^{2} y}{\tanh \left(e B L^{2} y\right)} a_{n}\left(\operatorname{tr}_{\mathcal{R}} \Omega^{n}+\text { c.c. }\right)-2\right\},
$$

where

$$
\Omega=e^{i L A_{0}^{a} T^{a}}
$$

is the Wilson line wrapping the $\mathbb{S}^{1}$ circle, and the pre-factor $a_{n}$ is

$$
a_{n}=\left\{\begin{array}{cc}
(-1)^{n} & \text { for thermal compactification } \mathbb{S}_{-}^{1}, \\
1 & \text { for spatial compactification } \mathbb{S}_{+}^{1} .
\end{array}\right.
$$

depending on the spin-connection of fermions over the $\mathbb{S}^{1}$ circle. Notice that in obtaining (2.9) we omitted the zero mode, $n=0$, which gives a divergent but otherwise holonomy independent contribution. ${ }^{5}$ We also note that the last term in (2.9) is independent of $B$ and $A_{0}^{a}$ and hence can be neglected in our subsequent analysis. Finally, upon using the change of variables $u=m^{2} L^{2} y$ in (2.9), we find

$$
\mathcal{V}^{\mathbb{R}^{3} \times \mathbb{S}_{ \pm}^{1}}=\frac{2}{\pi^{2} L^{4}} \sum_{n=1} \mathcal{M}_{n}^{2}(m, B) a_{n} \frac{\left(\operatorname{tr}_{\mathcal{R}} \Omega^{n}+\text { c.c. }\right)}{n^{4}},
$$

where the effective mass square term $\mathcal{M}_{n}^{2}(m, B)$ is given by

$$
\mathcal{M}_{n}^{2}(m, B)=\frac{z_{n}^{4}}{16} \int_{0}^{\infty} \frac{d u}{u^{3}} e^{-\frac{z_{n}^{2}}{4 u}-u} \frac{x u}{\tanh (x u)},
$$

and $z_{n}=n m L$, and $x=e B / m^{2}$. Equation (2.12) is our main result. The form of $\operatorname{tr}_{\mathcal{R}} \Omega^{n}+$ c.c. for the fundamental (F), adjoint (adj), symmetric (S) and anti-symmetric $(\mathrm{AS})$ representations is given by ${ }^{6}$

$$
\operatorname{tr}_{\mathcal{R}} \Omega^{n}+\text { c.c. }= \begin{cases}\operatorname{tr} \Omega^{n}+\operatorname{tr} \Omega^{* n}, & 1-\mathrm{F} \text { Dirac }, \\ \left|\operatorname{tr} \Omega^{n}\right|^{2}, & 1-\text { adj Weyl } \\ \frac{1}{2}\left[\left(\operatorname{tr} \Omega^{n}\right)^{2} \mp \operatorname{tr} \Omega^{2 n}\right]+\text { c.c. }, & 1-\text { AS } / \text { S Dirac } .\end{cases}
$$

\footnotetext{
${ }^{5}$ The $n=0$ term corresponds to the fermions vacuum correction in the presence in the magnetic field, and leads to charge renormalization which we ignore here.

${ }^{6}$ For $\mathrm{SU}(N)$ pure $\mathrm{YM}$ and $\mathrm{QCD}(\operatorname{adj})$, the center symmetry is $\mathbb{Z}_{N}$. For odd $N$, and $\mathcal{R}=\mathrm{F}, \mathrm{S} / \mathrm{AS}$, the center symmetry is trivial, $\mathbb{Z}_{1}$. For even $N, \mathcal{R}=\mathrm{F}, \mathrm{S} / \mathrm{AS}$, the center symmetry is $\mathbb{Z}_{1}$, and $\mathbb{Z}_{2}$. These global symmetries are also manifest in the one-loop potential.
} 
Notice that we give the result per Weyl fermion for the case of adjoint representation, keeping in mind that in this case we need an even number of Weyl fermions to avoid gauge anomaly. In the $B=0$ and $m=0$, and $B=0$ and $m \neq 0$, we obtain known results in thermal $[19,24-26]$ and spatial compactification $[26,43,44]$, also see $[45,46]$. In the large magnetic field limit, this expression reduces to

$$
\begin{aligned}
\mathcal{V}_{ \pm}[\Omega] \stackrel{\text { large-B }}{\longrightarrow} & \mathcal{V}_{\text {gauge }}^{\mathbb{R}^{3} \times S_{L}^{1}}[\Omega]+\left(\frac{|e B|}{2 \pi}\right) \mathcal{V}_{ \pm}^{\mathbb{R}^{1} \times S_{L}^{1}}[\Omega] \\
= & -\frac{2}{\pi^{2} L^{4}} \sum_{n=1} \frac{\left|\operatorname{tr} \Omega^{n}\right|^{2}}{n^{4}}+\left(\frac{|e B|}{2 \pi}\right) \frac{n_{f}}{\pi L^{2}} \sum_{n=1} \frac{(n L m) K_{1}(n L m)}{n^{2}}( \pm)^{n}\left(\operatorname{tr}_{\mathcal{R}} \Omega^{n}+\text { c.c. }\right) .
\end{aligned}
$$

Hence, in the presence of a strong magnetic field, the fermion contribution behaves as if fermions live on a space-time dimensionality $d-2=2$, i.e. on $\mathbb{R}^{1} \times \mathbb{S}^{1}$ instead of $\mathbb{R}^{3} \times \mathbb{S}^{1}$.

\section{Landau levels and the role of the lowest Landau level}

In this section, we express the one-loop potential (2.9) as a sum over all Landau levels. In particular, we show that the strong field limit (2.15) is solely due to the contribution of the lowest Landau level (LLL).

The spectrum of the Dirac operator on $\mathbb{R}^{3} \times \mathbb{S}^{1}$ in the presence of a non-trivial holonomy along the $\mathbb{S}^{1}$ direction and magnetic field $B$ perpendicular to $\mathbb{S}^{1}$ is given by

$$
\lambda_{\sigma, p, n, k_{z}, A_{0}}=m^{2}+k_{z}^{2}+\left(\omega_{n}+A_{0}^{a} T^{a}\right)^{2}+|e B|(2 p+1+\sigma),
$$

where $k_{z}$ is the momentum along the $z$-direction (perpendicular to both $\mathbb{S}^{1}$ and the $x-t$ plane), $\omega_{n}$ is the Kaluza-Klein frequency along the compact direction, $p=0,1,2, \ldots$ is the Landau level, $\frac{\sigma}{2}=\frac{ \pm 1}{2}$ is the spin. Every Landau level has $|e B| /(2 \pi)$ degeneracy factor for each spin alignment. Note that the LLL is given by $p=0, \sigma=-$, while the higher Landau levels also have additional pairing degeneracy between $(p+1, \sigma=-)$ and $(p, \sigma=+)$.

The zeta function associated with the Dirac operator is given by ${ }^{7}$

$$
\zeta_{\text {Dirac }}(s)=V_{\mathbb{R}^{3}} \frac{|e B|}{2 \pi} \sum_{p=0}^{\infty} \sum_{n \in \mathbb{Z}} \sum_{\sigma= \pm} \int \frac{d k_{z}}{2 \pi} \operatorname{tr}_{\mathcal{R}}\left[\left(\lambda_{\sigma, p, n, k_{z}, A_{0}}\right)^{-s}\right] .
$$

The fermion contribution to the one-loop potential $\mathcal{V}$ for the Wilson line holonomy on $\mathbb{R}^{3} \times \mathbb{S}^{1}$ can be extracted from this expression and is given by

$$
\mathcal{V}[\Omega]=-\log Z /\left(L V_{\mathbb{R}^{3}}\right)=\zeta^{\prime}(0) /\left(L V_{\mathbb{R}^{3}}\right),
$$

where the logarithm of partition function is

$$
\log Z=V_{\mathbb{R}^{3}} \frac{|e B|}{2 \pi} \sum_{p=0}^{\infty} \sum_{n \in \mathbb{Z}} \sum_{\sigma= \pm} \int \frac{d k_{z}}{2 \pi} \operatorname{tr}_{\mathcal{R}}\left[\log \lambda_{\sigma, p, n, k_{z}, A_{0}}\right] .
$$

\footnotetext{
${ }^{7}$ Recall that the determinant of an operator $\mathcal{O}$ with eigen-spectrum $\{\lambda\}$, i.e., $\mathcal{O} \psi_{\lambda}=\lambda \psi_{\lambda}$, is given by $\operatorname{Det} \mathcal{O}=\prod_{\lambda} \lambda=e^{\sum_{\lambda} \log \lambda}=e^{\operatorname{tr} \log \mathcal{O}}$, where $\{\lambda\}$ are the eigenvalues of the operator $O$. Using the definition of the zeta function, $\zeta(s)=\sum_{\lambda} \lambda^{-s}$, we find $\operatorname{Det} \mathcal{O}=\exp \left[-\zeta^{\prime}(s=0)\right]$.
} 
Before proceeding with this expression, it is also useful to make connection with the usual methods of statistical mechanics. The partition function of a free fermion gas in a magnetic field is $Z=\operatorname{tr}\left(e^{-\beta H}\right)=\prod_{Q} Z_{Q}=\prod_{Q}\left(1+e^{-\beta E_{Q}}\right)^{-1}$, where $Q=\left\{p, k_{z}, \sigma\right\}$ is a collective index for the quantum numbers of the states (defined above), and $\log Z=$ $-\sum_{Q} \log \left(1+e^{-\beta E_{Q}}\right)$. The energy eigenstates for a relativistic particle in a constant magnetic field is given by

$$
E_{k_{z}, p, \sigma}=\sqrt{m^{2}+k_{z}^{2}+|e B|(2 p+1+\sigma)} .
$$

Consequently, the partition function can be written as

$$
-\log Z=2 \operatorname{dim}(\mathcal{R}) V_{\mathbb{R}^{3}} \frac{|e B|}{2 \pi} \sum_{p=0}^{\infty} \sum_{\sigma= \pm} \int \frac{d k_{z}}{2 \pi} \log \left(1+e^{-\beta E_{k_{z}, p, \sigma}}\right) .
$$

If a Wilson line $\Omega=e^{i \beta A_{0}^{a} T^{a}}$ is turned on, this expression is modified into

$$
-\log Z=V_{\mathbb{R}^{3}} \frac{|e B|}{2 \pi} \sum_{p=0}^{\infty} \sum_{\sigma= \pm} \int \frac{d k_{z}}{2 \pi}\left[\operatorname{tr}_{\mathcal{R}} \log \left(1+e^{-\beta E_{k_{z}, p, \sigma}} \Omega\right)+\text { c.c. }\right]
$$

where the first term is due to quarks and the second term is due to anti-quarks. For trivial Wilson line background, i.e. $A_{0}^{a} T^{a}=0,(3.7)$ reduces to (3.6).

In the field theory expression (3.4), performing the sum over the Kaluza-Klein modes gives the statistical mechanics expression (3.7), and this reduces to (3.6) for trivial holonomy background.

Using the degeneracy $E_{k_{z}, p+1,-}=E_{k_{z}, p,+}$ for $p \geq 1$, we can perform the summation over spin $\sigma$, and rewrite (3.7) as a sum over the Landau levels, where the LLL appears once and $p \geq 1$ levels appear twice due to the aforementioned degeneracy.

$$
-\log Z=f(m)+2 \sum_{p=1}^{\infty} f\left(m_{p}\right), \quad m_{p} \equiv\left[m^{2}+2|e B| p\right]^{1 / 2},
$$

where $m_{p}$ is effective mass associated with level $p$. The functional form of the contribution of the LLL and higher LLs are the same, and is given by

$$
\begin{aligned}
f(m) & =V_{\mathbb{R}^{3}} \frac{|e B|}{4 \pi^{2}} \int_{-\infty}^{\infty} d k_{z} \operatorname{tr}_{\mathcal{R}} \log \left(1+e^{-\beta \sqrt{k_{z}^{2}+m^{2}}} \Omega\right)+\text { c.c. } \stackrel{k_{z}=m \sinh t, \text { Taylor expand } \log }{\longrightarrow} \\
& =V_{\mathbb{R}^{3}} \frac{|e B|}{2 \pi^{2}} m \int_{0}^{\infty} d t \cosh t \sum_{n=1}^{\infty} \frac{(-1)^{n+1}}{n} e^{-n \beta m \cosh t}\left(\operatorname{tr}_{\mathcal{R}} \Omega^{n}+\text { c.c. }\right) \\
& =V_{\mathbb{R}^{3}} \frac{|e B|}{2 \pi^{2}} m \sum_{n=1}^{\infty} \frac{(-1)^{n+1}}{n} K_{1}(m \beta n)\left(\operatorname{tr}_{\mathcal{R}} \Omega^{n}+\text { c.c. }\right) .
\end{aligned}
$$

It is straightforward to repeat the same steps for fermions endowed with periodic boundary conditions. As a result, the fermion induced potential for the Wilson line can be 
expressed as

$$
\begin{aligned}
\mathcal{V}^{ \pm}= & \mathcal{V}_{\mathrm{LLL}}^{ \pm}+2 \sum_{p=1}^{\infty} \mathcal{V}_{\mathrm{p}^{\mathrm{th}}-\mathrm{LL}}^{ \pm} \\
= & \left(\frac{|e B|}{2 \pi}\right) \frac{1}{\pi L^{2}} \sum_{n=1}^{\infty} \frac{( \pm)^{n}}{n^{2}}(m L n) K_{1}(m L n)\left(\operatorname{tr}_{\mathcal{R}} \Omega^{n}+\text { c.c. }\right) \\
& +\left(\frac{|e B|}{\pi}\right) \sum_{p=1}^{\infty} \frac{1}{\pi L^{2}} \sum_{n=1}^{\infty} \frac{( \pm)^{n}}{n^{2}}\left(m_{p} L n\right) K_{1}\left(m_{p} L n\right)\left(\operatorname{tr}_{\mathcal{R}} \Omega^{n}+\text { c.c. }\right) .
\end{aligned}
$$

The leading term $\mathcal{V}_{\mathrm{LLL}}^{ \pm}$is exactly the fermion induced term in (2.15). Since the energy of the LLL, $E_{-, p=0, k_{z}}$, is $B$ independent, the linear behavior with $B$ comes only from the density of states. The terms with $p \geq 1$ are the contributions from the higher Landau levels. Note that apart from the factor of two difference with respect to the LLL contribution coming from the spectral degeneracy, the functional form of these contributions are the same as the LLL with the replacement $m \rightarrow m_{p}=\left[m^{2}+2|e B| p\right]^{1 / 2}$, where $m_{p}$ is an effective mass of quarks associated with level $p$.

In the large- $B$ limit, the contributions coming from higher Landau levels are exponentially suppressed, for example, $K_{1}\left(m_{p} L n\right) / K_{1}(m L n) \sim e^{-L \sqrt{2 p|e B|+1}}$, and we obtain (2.15).

Equivalence of (2.9) and (3.10). To see this, we start with (2.9) and use the identity

$$
\frac{1}{\tanh x}=1+2 \sum_{p=1}^{\infty} e^{-2 p x} .
$$

This helps us to express (2.9) as a summation over all Landau levels. Writing the fermion induced potential as $\sum_{n=1}^{\infty} I^{(n)}( \pm)^{n}\left(\operatorname{tr}_{\mathcal{R}} \Omega^{n}+\right.$ c.c. $)$, we have

$$
\begin{aligned}
I^{(n)} & =\frac{2}{(4 \pi)^{2} L^{4}} \int_{0}^{\infty} \frac{d y}{y^{3}} e^{-\frac{n^{2}}{4 y}-m^{2} L^{2} y} \frac{e B L^{2} y}{\tanh \left(e B L^{2} y\right)} \\
& =\frac{|e B|}{8 \pi^{2} L^{2}}\left(\int_{0}^{\infty} \frac{d y}{y^{2}} e^{-\frac{n^{2}}{4 y}-m^{2} L^{2} y}+2 \sum_{p=1}^{\infty} \int_{0}^{\infty} \frac{d y}{y^{2}} e^{-\frac{n^{2}}{4 y}-\left(m^{2}+2 p|e B|\right) L^{2} y}\right) \\
& =\left(\frac{|e B|}{2 \pi}\right) \frac{1}{\pi L^{2}} \frac{n L m K_{1}(n L m)}{n^{2}}+\left(\frac{|e B|}{\pi}\right) \sum_{p=1}^{\infty} \frac{1}{\pi L^{2}} \frac{n L m_{p} K_{1}\left(n L m_{p}\right)}{n^{2}} \\
& =I_{0}^{(n)}+2 \sum_{p=1}^{\infty} I_{p}^{(n)},
\end{aligned}
$$

which is a sum over all Landau levels, equal to (3.10).

\subsection{Magnetic susceptibility and its jump across the deconfinement transition}

The magnetic susceptibility is a measure of the response of the QCD thermal equilibrium state (or ground state) to an external magnetic field (see for example [13, 47].) Here, we identify the jump in magnetic susceptibility as an order parameter for the confinement/deconfinement phase transition. We consider the magnetic susceptibility first for thermal and then for spatial compactification of QCD-like theories. 
Thermal compactification. Denote the free energy density of QCD as a function of magnetic field and inverse temperature as $\mathcal{F}(B, \beta)=-\frac{1}{\beta V^{3} 3} \log Z(B, \beta)$ where $Z$ is the thermal partition function. We define the magnetic susceptibility as:

$$
\xi=-\left.\frac{\partial^{2} \mathcal{F}}{\partial(e B)^{2}}\right|_{B=0} .
$$

The free energy can be calculated in two related ways. One is by simply extremizing the one-loop potential with respect to holonomy, and the other is by using methods of statistical mechanics. Both yield the same result.

In the high-temperature deconfined phase, the minimum of the one-loop potential is located at $\Omega=1$ and consequently we can use (2.9), keeping in mind that $\mathcal{F}=\mathcal{V}^{\mathbb{R}^{3} \times \mathbb{S}_{-}^{1}}$, to find

$$
\begin{aligned}
\xi\left(\beta<\beta_{c}\right) & =\frac{1}{3 \pi^{2}}\left[\sum_{n=1}^{\infty} K_{0}(m n \beta)(-1)^{n+1}\right] \operatorname{dim}(\mathcal{R}) \\
& =\frac{1}{3 \pi^{2}}\left[\int_{0}^{\infty} d t \frac{1}{e^{m \beta \cosh t}+1}\right] \operatorname{dim}(\mathcal{R}) \approx \begin{cases}O\left(N^{1}\right) & \mathcal{R}=F \\
O\left(N^{2}\right) & \mathcal{R}=\mathrm{AS} / \mathrm{S} / \mathrm{Adj}\end{cases}
\end{aligned}
$$

where $\beta_{c} \sim \Lambda^{-1}$ is the strong length scale. Clearly, $\xi>0$ and the deconfined phase is paramagnetic. Also, we find that (3.14) is compatible with the large- $N$ scaling of liberated quarks and their free energy.

For the low temperature confined phase, we cannot calculate the magnetic susceptibility due to strong coupling. However, there exists a semi-classically calculable deformation of QCD and YM theory which is continuously connected to confining low temperature regime $[36,55]$. Multiple non-perturbative aspects of deformed QCD confirming the continuity idea are studied in continuum [48-52] and in lattice [53, 54]. The main idea is to deform Yang-Mills theory with a center-stabilizing double-trace operator on small $\mathbb{S}^{1} \times \mathbb{R}^{3}$ such that the minimum of the potential is at a center-symmetric point. For example, for the defining representation, the minimum of the potential is at $\Omega=\eta_{N} \operatorname{Diag}\left(1, e^{i \frac{2 \pi}{N}}, e^{i \frac{4 \pi}{N}}, \ldots, e^{i \frac{2 \pi(N-1)}{N}}\right)$, where $\eta_{\text {odd }}=1$ and $\eta_{\text {even }}=e^{i \frac{\pi}{N}}$, as shown in figure 1c for $\mathrm{SU}(2)$ gauge group. (See section 5 for the relation between the strong and weak coupling center-symmetric regimes.)

In the weak coupling abelian confinement regime, the one-loop induced potential for fermions is still (2.9), but the implication is now different. The reason is that introducing fundamental fermions in the weak coupling confinement regime of deformed Yang-Mills distorts center-symmetric vacuum only slightly. In fact, the trace of the Wilson line changes as $\frac{1}{N} \operatorname{tr} \Omega=0 \rightarrow \frac{1}{N} \operatorname{tr} \Omega=O\left(N^{-1}\right)$, i.e. the theory almost respects center symmetry. In the framework of deformed-QCD, which provides a weak coupling continuation of the confined phase, we can calculate the sign and $N$ scaling of the magnetic susceptibility. Since the center-symmetry is preserved the quarks are confined in color-singlet states, and therefore we find $\xi=O\left(N^{0}\right)>0$ and the theory is in a paramagnetic phase. It is reasonable to assume that this result in weak coupling abelian confinement regime extrapolates to strong coupling non-abelian confinement regime. In fact, $O\left(N^{0}\right)$ magnetic susceptibility is 
in accordance with the fact that the spectral density of the color singlet states (and free energy density) in the confined phase is $O\left(N^{0}\right)$. More explicitly, working with a hadron resonance gas model [56] in the large- $N$ limit, we obtain a susceptibility of order $O\left(N^{0}\right)$. Therefore, the $N$ scaling differs quantitatively between the deconfined and confined phases:

$$
\xi(\beta)= \begin{cases}O\left(N^{1}\right) \text { or } O\left(N^{2}\right) & \beta<\beta_{c}, \\ O\left(N^{0}\right) & \beta>\beta_{c},\end{cases}
$$

for one-index and two-index representation fermions, respectively. The jump in the magnetic susceptibility provides an order parameter for deconfinement phase transition. This jump agrees very well with recent lattice studies [47].

Spatial compactification. We can also study the response of the spatially compactified theory to external magnetic field. Define the "twisted susceptibility" in the zero temperature, but spatially compactified theory, as

$$
\xi^{\mathrm{tw}}=-\frac{\partial^{2}}{\partial(e B)^{2}}\left(-\frac{1}{L V_{\mathbb{R}^{3}}} \log Z_{+}(B, L)\right) .
$$

For $\mathcal{R}=\mathrm{F} / \mathrm{AS} / \mathrm{S}$ in the small- $L$ regime, $L<L_{c}$ and $L_{c} \sim \Lambda^{-1}$, where spatial (approximate) center symmetry is spontaneousy broken, we have $\xi^{\mathrm{tw}} \sim O\left(N^{1}\right)$ for $\mathrm{F}$ and $O\left(N^{2}\right)$ for AS/S, and the susceptibility is negative $\xi^{\mathrm{tw}}<0$, i.e. the phase $L<L_{c}$ is diamagnetic. On the other hand, for $L>L_{c}$ we have approximate center symmetry, assuming large $N$ and keeping $n_{f}$ small, and hence the quarks form singlets and we have $\xi^{\mathrm{tw}} \sim O\left(N^{0}\right)<0$. Note that the signs of susceptibilities are opposite for the thermal versus spatial compactification for complex representations, but the $N$ scaling of $\xi^{\text {tw }}$ is the same as the regular susceptibilities (3.15). For $\mathcal{R}=$ Adj with periodic boundary conditions, there is no center symmetry changing phase transition for sufficiently light fermions, and $\xi \sim O\left(N^{0}\right)$ at any $L$. We explore this case in the next section.

\section{Massive QCD(adj) in external magnetic field}

By inspecting the fermion induced one-loop potential (2.12) and (2.14), it is not hard to see that the center symmetry is broken for all representations $\mathcal{R}$ except for the adjoint representation of $\mathrm{SU}(N)$ with periodic boundary conditions (spatial compactification). QCD with $n_{f}$ adjoint Weyl fermions, QCD(adj), possesses a classical global chiral $\mathrm{SU}\left(n_{f}\right) \times \mathrm{U}(1)$ symmetry. The $\mathrm{U}(1)$ symmetry is anomalous and reduces down to $\mathbb{Z}_{2 N n_{f}}$ due to instanton effects. Below, we restrict attention to $n_{f}=2$, in which case the global symmetry is just $\mathrm{SU}(2) \times \mathbb{Z}_{4 N}$.

To couple the system to a $\mathrm{U}(1)$ magnetic field, we gauge a $\mathrm{U}(1)$ subgroup of the flavor $\mathrm{SU}(2)$. This $\mathrm{U}(1)$ subgroup is taken to be of the diagonal form diag $(1,-1)$. This amounts to assigning opposite charges to the two different flavors which in turn guarantees the absence of gauge anomalies. In addition, requesting QCD(adj) to be an asymptotically free theory, we find that $n_{f}$ has to be either 2 or 4 . At small compactification radius, $N L \Lambda / 2 \pi \lesssim 1$ where $\Lambda$ is the strong coupling scale, the Kaluza-Klein modes as well as 
the modes which carry a fraction of the KK-momentum are weakly coupled and can be integrated out perturbatively. Hence, the one-loop potential resulting from integrating out the non-zero Kaluza-Klein modes of the gauge field and $n_{f}$ Weyl fermions with mass $m$ reads, in the limit of large-magnetic fields,

$$
\mathcal{V}_{+}^{\mathbb{R}^{3} \times S_{L}^{1}}[\Omega]=\frac{2}{\pi^{2} L^{4}} \sum_{n=1} \mathcal{M}_{n}^{2}(m, B) \frac{\left|\operatorname{tr} \Omega^{n}\right|^{2}}{n^{4}},
$$

with effective mass (square) for the Wilson line

$$
\mathcal{M}_{n}^{2}=-1+\frac{n_{f}}{4} x z_{n}^{3} K_{1}\left(z_{n}\right), \quad z_{n}=n m L, \quad x=\frac{|e B|}{m^{2}} .
$$

The traces $\operatorname{tr} \Omega^{n}$, with $n=\lfloor N / 2\rfloor$ where $n=\lfloor\cdot\rfloor$ is the (lower) floor function, are independent variables. Therefore, if the effective mass square $\mathcal{M}_{n}^{2}$ are positive for all $n \leq\lfloor N / 2\rfloor$, then the $\mathbb{Z}_{N}$ center symmetry is unbroken with $\operatorname{tr} \Omega^{n}=0$ for all $n \neq 0 \bmod N$. If $\mathcal{M}_{1}^{2}, \mathcal{M}_{2}^{2}, \ldots$ are negative, then the center symmetry is completely broken. If some of the masses are tachyonic, then a subgroup of $\mathbb{Z}_{N}$ center symmetry breaks down spontaneously, for details see [37].

The vanishing of the effective mass square for $x=\{0,1,5,10\}$ occurs at $z_{n}^{*}=$ $\{2.07,2.39$,

$5.44,6.61\}$ for $n_{f}=2$, and at $z_{n}^{*}=\{3.16,3.39,6.61,7.67\}$ for $n_{f}=4$. Since $z_{n}^{*}$ increases with increasing the field strength, a strong field will stabilize the center symmetry for larger values of the compact dimension $L$ at fixed $m$. In effect, this reduces the center-symmetric breaking zone as illustrated in figure 1. In the formal infinite magnetic field limit, the center-breaking phase disappears completely for any fixed value of the fermion mass $m$.

\subsection{Abelian confinement and large- $N$ volume independence regimes}

In the absence of magnetic field, a general $\mathrm{SU}(N)$ gauge theory with sufficiently light adjoint fermions, $m<m^{*} \sim \Lambda$, (as shown on the left panel of figure 1 for $\mathrm{SU}(2)$ ) endowed with periodic boundary condition is center-symmetric at any value of the compatification radius $L:\left\langle\operatorname{tr} \Omega^{n}\right\rangle=0, n \neq 0(\bmod N)$ and exhibits continuity in the sense of center-symmetry. As shown in figure 2, unbroken center symmetric holonomy has different implications depending on whether the theory is weakly or strongly coupled. See next section for more details. A QCD-like theory remaining center-symmetric at any compactification radius has two extreme regimes:

- $N L \Lambda / 2 \pi \gg 1$ : non-abelian confinement, volume independence (at large $N$ ) regime.

- $N L \Lambda / 2 \pi \ll 1$ : abelian confinement, adjoint Higgsing (or Hosotani regime).

The associated Wilson line holonomies are shown in figure $2 \mathrm{~A}$ and $\mathrm{C}$.

In the absence of magnetic field and for $m>m^{*} \sim \Lambda$, there are three regions as shown in the left panel of figure 1 for $\mathrm{SU}(2)$. At sufficiently small- $L$, given by $N L m \lesssim z^{*}$ for $\mathrm{SU}(N)$ (typical values of $z^{*}$ are given in the previous section), the $\mathbb{Z}_{N}$ center symmetry restores completely [37]. With mass $m \gtrsim \Lambda$, the small- $L$ center-symmetric regime in the lower left 
corner of the left panel of figure 1 corresponds to $N L \Lambda \lesssim 1$, where $L \sim O\left(N^{-1}\right)$, and is not the volume independence domain, but rather volume dependent abelian-confinement domain. On the other hand, for $m \gtrsim \Lambda$ and for $N L \Lambda \gtrsim 1$ (at large $N$ ) we have a large- $L$ center-symmetric, non-abelian confinement and volume independent regime. These two regimes $(N L \Lambda \lesssim 1$ and $N L \Lambda \gtrsim 1)$ are separated by an intermediate phase in which centersymmetry is spontaneously broken as shown in figure 1 for $\mathrm{SU}(2)$.

This phase separation between the large and small $L$ regimes can be avoided in the presence of a strong magnetic field since the field sets a new scale which parametrically enhances the effect of the adjoint fermions. In the presence of a very large magnetic field, the condition for the preservation of center-symmetry is $N L m<z^{*}\left(\frac{|e B|}{m^{2}}\right)$ (typical values of $z^{*}$ in the presence of strong field are given in the previous section). Thus, the hierarchy

$$
\sqrt{e B} \gg m>\Lambda
$$

can help the stabilization of the center symmetry at larger values of $L$, reducing the region in which the center is broken. In particular, in the (formal) exponentially large- $B$ field limit, (such that it can undo the effect of the mass term for fermions) the intermediate regime in which center-symmetry is broken shrinks, and gradually disappears. Consequently, the infinite- $B$ theory with any finite fermion mass $m$ and large $N$ possesses both volume independent non-abelian confinement regime $N L \Lambda / 2 \pi \gg 1$ and volume dependent abelian confinement regime $N L \Lambda / 2 \pi \lesssim 1$, as we vary $L$. In particular, the center symmetry is always respected and it has a double life: one at small $L$ (weak coupling), and the other at large $L$ (strong coupling).

For the purpose of the center-symmetry preservation in the weak coupling regime, this is a non-decoupling of large mass fermion, (due to its enhancement by LLL density of states), and the center-symmetry stabilizes. However, in the same time the low energy effective field theory (the dynamics at distances larger than $1 / m$ ) is a pure YM theory up to $\Lambda / m$ corrections! $!^{8}$

\footnotetext{
${ }^{8} \mathrm{We}$ also comment on the fate of center-symmetry in the light of the results of [57]. In this work, the authors argue that a quark dynamical mass can be generated in QCD on $\mathbb{R}^{4}$ in external magnetic field, thanks to magnetic catalysis. A self-consistent solution to the Schwinger-Dyson equation leads to the dynamical quark mass expression:

$$
m_{q}^{2} \sim|e B| \alpha_{s}^{2 / 3} \exp \left[-\frac{4 \pi N}{\alpha_{s}\left(N^{2}-1\right) \log \left(1 / \alpha_{s}\right)}\right],
$$

where the strong coupling $\alpha_{s} \equiv \frac{g^{2}}{4 \pi}$ is related to the scale $|e B|$ as $\alpha_{s}=\frac{12 \pi}{11 N-2 n_{f} N} \frac{1}{\log \left(e B / \Lambda^{2}\right)}$. In addition, the gluon acquires a mass $M_{g}$ due to a large fermionic contribution to the polarization operator: $M_{g}^{2} \sim \alpha_{s}|e B|$. At magnetic length scales much smaller than any scale in $\mathrm{QCD}(\operatorname{adj})$ on $\mathbb{R}^{3} \times \mathbb{S}^{1}$, i.e. for $\sqrt{|e B|} \gg L^{-1} \gg \Lambda$, our theory lives on $\mathbb{R}^{4}$ or in other words we can completely neglect the fact that one of the spacial dimensions is compactified. In this case, we can express the quarks and the gluon masses in terms of $|e B| / \Lambda$ as

$$
\frac{|e B|}{M_{q}^{2}} \sim\left[\log \left(\frac{|e B|}{\Lambda^{2}}\right)\right]^{2 / 3}\left(\frac{|e B|}{\Lambda^{2}}\right)^{\frac{3 N^{2}\left(11-2 n_{f}\right)}{N^{2}-1}}, \frac{|e B|}{M_{g}^{2}} \sim \log \frac{|e B|}{\Lambda^{2}} .
$$

We see that in the limit $\sqrt{|e B|} \gg \Lambda$, both $\frac{|e B|}{M_{q}^{2}}$ and $\frac{|e B|}{M_{g}^{2}} \rightarrow \infty$. Upon calculating the effective potential on $\mathbb{R}^{3} \times \mathbb{S}^{1}$, we can use (4.1) but now taking into account the fact that the quarks and gluon are massive.
} 


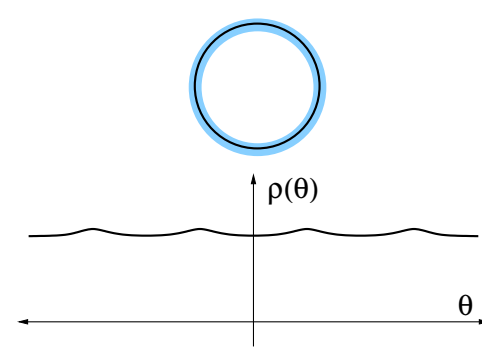

A) Unbroken (non-abelian)

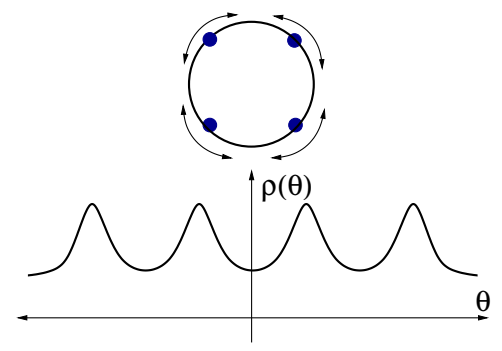

B)Intermediate

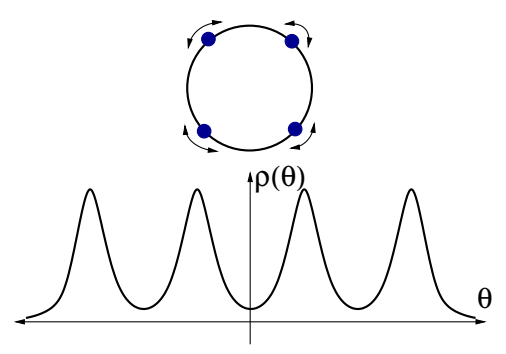

C)Adjoint-Higgsing (abelianization)

Figure 2. Realizations of unbroken center symmetry $\langle\operatorname{tr} \Omega\rangle=0$ from strong to weak coupling, both in continuum and in lattice. A) Strong coupling non-trivial holonomy, eigenvalues are randomized over the eigenvalue circle. B) Intermediate coupling. C) Weak coupling non-trivial holonomy. Eigenvalues are at the roots of unity (up to a phase) and their fluctuations are small. These regimes are continuously connected in the sense of center symmetry, C) is non-perturbatively calculable.

\section{Comments on lattice realization of abelian confinement and Hosotani mechanism on $\mathbb{R}^{3} \times S^{1}$}

As already mentioned, it is crucial to emphasize that unbroken center symmetry with

$$
\left\langle\operatorname{tr} \Omega^{n}\right\rangle=0, \quad n \neq 0(\bmod N) \quad \text { at any } L
$$

has multiple different realizations depending on whether the theory is weakly or strongly coupled. This difference is not sufficiently addressed in literature, the first discussion of it is in [36] and a more through discussion can be found in section 5 of [58]. Our goal is not to repeat the same argument here, but rather to point out the lattice realization of the regimes shown in figure 2 :

- $N L \Lambda / 2 \pi \gg 1$ : Strong coupling non-trivial holonomy, gauge symmetry unbroken, figure $2 \mathrm{~A}$

- $N L \Lambda / 2 \pi \ll 1$ : Weak coupling non-trivial holonomy, gauge symmetry broken, figure $2 \mathrm{C}$

In the strong coupling regime, eigenvalues are randomized over the dual circle. This configuration cannot be viewed as a minimum of a potential in a local effective field theory, i.e, there is no parametric separation of scales that justify an effective field theory. In this regime, the average Wilson line determines the free energy. This is opposite to what $\overline{\text { Hence, we replace (4.2) with } \mathcal{M}_{n}^{2}=-\mathcal{M}_{n \mathrm{ph}}^{2}}+\mathcal{M}_{n \text { quark }}^{2}$, where

$$
\mathcal{M}_{n \mathrm{ph}}^{2} \sim-\frac{|e B|}{M_{g}^{2}}\left(n L m_{g}\right)^{3} \mathrm{~K}_{1}\left(n L m_{g}\right), \quad \mathcal{M}_{n q u a r k}^{2} \sim n_{f} \frac{|e B|}{M_{q}^{2}}\left(n L m_{q}\right)^{3} \mathrm{~K}_{1}\left(n L m_{q}\right) .
$$

However, inspection of the masses (4.5) reveals that the ratio $\frac{|e B|}{M_{q}^{2}}$ has a much stronger dependence on the magnetic field than $\frac{|e B|}{M_{g}^{2}}$. Hence, we find that for $B \rightarrow \infty$, the effective square mass $\mathcal{M}_{n}^{2}=-\mathcal{M}_{n \mathrm{ph}}^{2}+\mathcal{M}_{n \text { quark }}^{2}$ is always a positive quantity and the center-symmetry is preserved. This justifies our one-loop effective potential calculations throughout this paper. 
happens in the weak coupling (abelian) confinement regime where Wilson line potential can be viewed as a potential in a local effective field theory (with appropriate parametric separation of scales).

In center-symmetric weak coupling regime, (5.1) implies that the minimum of the one-loop potential for the Wilson line is at

$$
\Omega=\eta_{\mathrm{N}}\left(\begin{array}{cccc}
1 & & & \\
& e^{i \frac{2 \pi}{N}} & & \\
& & e^{i \frac{4 \pi}{N}} & \\
& & \ddots & \\
& & & e^{i \frac{2 \pi(N-1)}{N}}
\end{array}\right), \quad \text { where }\left\{\begin{array}{l}
\eta_{\text {odd }}=1 \\
\eta_{\text {even }}=e^{i \frac{\pi}{N}}
\end{array}\right.
$$

as shown in figure 1c for $\mathrm{SU}(2)$ and figure $2 \mathrm{C}$ for $\mathrm{SU}(4)$ gauge groups. In this regime, because of the weak coupling, the fluctuation of the eigenvalues are small and the theory, to all orders in perturbation theory, undergoes adjoint Higgsing, i.e. the long distance theory abelianizes:

$$
\mathrm{SU}(N) \rightarrow \mathrm{U}(1)^{N-1}
$$

The abelianized regime is realized if the theory is weakly coupled at the scale of the inverse of the lightest W-boson mass, $m_{W}^{-1}=L N / 2 \pi$ for the center-symmetric background [36], ${ }^{9}$

$$
\frac{g^{2} N(L N)}{4 \pi} \ll 1 \quad \text { or } \quad \frac{L N \Lambda}{2 \pi} \lesssim 1
$$

Whether the gauge fluctuations (photons of $\mathrm{U}(1)^{N-1}$ ) which are massless to all orders in perturbation theory acquire a dynamical mass or not depends on the details of the theory. In deformed-YM, the photons acquire a mass via monopole-instanton mechanism [36], and in $\mathcal{N}=1 \mathrm{SYM}$ and $\mathrm{QCD}(\operatorname{adj})$, they do so via the magnetic bion mechanisms [33]. However, in $\mathcal{N}=2 \mathrm{SYM}$ in center-symmetric background (5.2) in its Coulomb branch associated with Wilson line, the photons do not acquire a dynamical mass. Despite the fact that monopole-instantons do exist, their fermion zero mode structure and $\mathcal{N}=2$ extended supersymmetry does not permit the generation of mass gap [59]. The weak coupling regime provides an example of gauge symmetry breaking (or Hosotani mechanism) to all orders in perturbation theory for deformed $\mathrm{YM}$ and $\mathrm{QCD}(\operatorname{adj})$, and a non-perturbative realization of gauge symmetry breaking in the $\mathcal{N}=2$ SYM. In the deformed YM and QCD(adj), the IR-theory acquires a mass gap for gauge fluctuations, while in the $\mathcal{N}=2 \mathrm{SYM}$, the IR theory is gapless $\mathrm{U}(1)^{N-1}$ theory non-perturbatively.

The realization of the abelianization regime in lattice gauge theory requires the mapping of the regime (5.4) to lattice units. To emulate $\mathbb{R}^{3} \times \mathbb{S}^{1}$, consider a $4 \mathrm{~d}$ lattice $\Lambda_{4}$ with size $L_{1}=\Gamma_{1} \mathfrak{a}=L_{2}=\Gamma_{2} \mathfrak{a}=L_{3}=\Gamma_{3} \mathfrak{a} \gg L_{4}=\Gamma_{4} \mathfrak{a}$ where $\Gamma_{\mu}$ is the number of sites in a

\footnotetext{
${ }^{9}$ If $N=$ few, the appearance of $N$ in (5.4) hardly matters. However, in the large- $N$ limit, the correct combination determining if a center-symmetric theory is weakly coupled or not is $\frac{L N \Lambda}{2 \pi}$.
} 
given direction, and $\mathfrak{a}$ is lattice spacing. This is an asymmetric discretized 4 -torus. Define lattice gauge action with adjoint fermions as

$$
S[U]=\beta \sum_{p \in \Lambda_{4}} \frac{1}{N}\left(\operatorname{tr} U[\partial p]+\operatorname{tr} U^{\dagger}[\partial p]\right)+S_{\text {fermion }}, \quad \text { where } \beta=\frac{g_{0}^{2} N}{4 \pi},
$$

and $g_{0}^{2}=g^{2}(\mathfrak{a})$ is the bare coupling constant at the lattice cut-off scale $\mathfrak{a}$. In order to achieve abelianization of the long distance dynamics, one needs weak coupling at the scale $L_{4} N=N \Gamma_{4} \mathfrak{a}$,

$$
\frac{g^{2} N\left(N \Gamma_{4} \mathfrak{a}\right)}{4 \pi} \ll 1 \quad L_{4} \equiv \Gamma_{4} \mathfrak{a}=\text { fixed, as } \Gamma_{4} \rightarrow \infty, \mathfrak{a} \rightarrow 0
$$

Once this is achieved, the dynamics abelianizes at distances larger than the inverse lightest W-boson mass, where

$$
m_{W}=\frac{2 \pi}{L N} \quad \text { in continuum, } \quad m_{W}=\frac{2}{\mathfrak{a}} \sin \frac{\pi}{\Gamma_{4} N} \quad \text { in lattice. }
$$

For two point connected correlators, $\langle O(\mathbf{x}) O(\mathbf{0})\rangle$, in order to disentangle the short distance degrees of freedom from the long-distance $\mathrm{U}(1)^{N-1}$ photon modes, one needs (along the non-compact directions) separations larger than $|\mathbf{x}| \gtrsim \frac{\Gamma_{4} N \mathfrak{a}}{2 \pi}$. Therefore, to see the abelianized dynamics of the gapless photons (in perturbation theory), one must have

$$
L_{i}=\Gamma_{i} \mathfrak{a} \gtrsim \frac{\Gamma_{4} N \mathfrak{a}}{2 \pi}=\frac{L_{4} N}{2 \pi} \quad \text { abelianized (Hosotani) regime. }
$$

This may be considered as the Hosotani regime of the lattice gauge theory formulated on $T^{3} \times S^{1}$. It is extremely important to note that $L_{i} \gtrsim L_{4}$ is not sufficient to see the Hosotani regime. The decoupling of the non-Cartan sub algebra degrees of freedom i.e., W-bosons, occurs at scales larger than $m_{W}^{-1} \sim L_{4} N$. In particular, at large- $N$ limit, the abelianization only occurs at $L_{i}=\infty$ regardless of how small $L_{4}$ is so long as it is $O\left(N^{0}\right)$.

\subsection{The resolution of Eguchi-Kawai versus Hosotani puzzle}

Both Hosotani mechanism and Eguchi-Kawai demands the very same unbroken center symmetry condition in $\mathrm{QCD}(\mathrm{adj})$, yet they are completely different physical phenomenon. This is what we mean by Eguchi-Kawai versus Hosotani puzzle.

The overall picture and resolution should now be clear. In a working Eguchi-Kawai reduction, center symmetry does not break, and consequently, in the large- $N$ limit, gauge symmetry never breaks regardless of how small $L_{4}$ is so long as it is $O\left(N^{0}\right)$. In Hosotani mechanism, center symmetry does not break either, and yet gauge symmetry breaks at sufficiently weak coupling, which, in the large $N$ limit, scales as $L_{4} \sim O\left(N^{-1}\right)$. This is how i) abelianized (Hosotani) regime where gauge symmetry is broken, ii) non-abelian volume independence (Eguchi-Kawai) regime and iii) non-abelian large- $L$, finite- $N$ regimes where gauge symmetry remains unbroken mutually exclude each other without leading to any contradiction.

This intricate working of the physical scales is also most likely the reason that the research along these two directions (despite relying on the same physical condition of unbroken center symmetry) remained mutually exclusive so far. Clearly, without careful deliberation of scales they are in apparent conflict with each other. 


\subsection{How large should the box be in order to see the setting of mass gap and abelian confinement?}

Non-perturbatively, we also know that the photons on $\mathbb{R}^{3} \times \mathbb{S}^{1}$ acquire a mass gap in deformed YM and QCD(adj) with heavy fermions via monopole-instanton mechanism and in $\mathrm{QCD}$ (adj) with massless or light fermions via the magnetic bion mechanism $[33,36]$. This gap is, for example, in weak coupling deformed YM or center-symmetric regime of massive $\mathrm{QCD}(\mathrm{adj})$ is given by $m_{\mathrm{gap}}=m_{W} e^{-\frac{4 \pi^{2}}{g^{2}\left(m_{W}\right) N}}=\Lambda(\Lambda L N)^{5 / 6}$ where $\Lambda$ is the strong scale of YM theory.

In order to see the gap for the (dual) photons, the box size must also be larger than the inverse of the mass gap; otherwise one will always erroneously conclude that the theory is gapless. ${ }^{10}$ This requires

$$
L_{i} \gtrsim m_{\text {gap }}^{-1}=m_{W}^{-1} e^{+\frac{4 \pi^{2}}{g^{2}\left(m_{W}\right) N}}=\frac{L_{4} N}{2 \pi} e^{+\frac{4 \pi^{2}}{g^{2}\left(m_{W}\right) N}} . \quad \text { abelian confinement regime }
$$

Admittedly, it may be difficult to achieve such a hierarchy in practical simulations and also hard to see the regime of abelian confinement, but we are not pessimists on this, and there is a very strong incentive to pursue this direction, see section 5.3.

On a practical side, on a $\Gamma_{i}^{3} \times \Gamma_{4}=16^{3} \times 4$ lattice formulation of $\mathrm{SU}(3)$ lattice $\mathrm{QCD}(\mathrm{adj})$ with $m \mathfrak{a}=0.1$ where $m$ is bare quark mass, the small- $L$ confined phase is achieved at $\beta>\beta^{*}=6.30$ [42]. Making, for example, $\beta \gtrsim 10$, forcing the theory to remain weakly coupled at $N \Gamma_{4} \mathfrak{a}$, one can certainly achieve abelianization. But making $\beta$ so large also makes the length scale of the mass gap $m_{\text {gap }}^{-1}$ much larger than box-size $\Gamma_{i} \mathfrak{a}$ along the large dimensions. This, as explained above, will lead to the incorrect conclusion that the theory is gapless. Thus, one needs to make $\Gamma_{i}$ as large as possible and make $\beta>\beta^{*}$ as small as possible while remaining in abelianized regime.

\subsection{Why is the weak coupling corner important both for lattice and continuum studies?}

There are only a handful of theories in which confinement and mass gap can be understood by reliable field theory methods in three and four dimensions. These are:

- Softy broken $\mathcal{N}=2$ SYM theory down to $\mathcal{N}=1$ SYM on $\mathbb{R}^{4}$ and $\mathbb{R}^{3} \times \mathbb{S}^{1}[59]$,

- Polyakov model on $\mathbb{R}^{3}[60]$

- $\mathrm{QCD}(\operatorname{adj})[33]$ and deformed YM [36] on small $\mathbb{R}^{3} \times \mathbb{S}^{1}$.

It is currently not feasible to simulate softy broken $\mathcal{N}=2 \mathrm{SYM}$ theory on lattice despite much progress in lattice supersymmetry. It is also technically very difficult to simulate Polyakov model on $\mathbb{R}^{3}$, due to fine tunings (for scalar masses and quartics, for

\footnotetext{
${ }^{10}$ This is the main danger with lattice simulations of the abelian confinement regime. Although one can see (by current techniques) both abelianization (Hosotani regime) and gapless photons, since no dramatic hierarchies are required to achieve this but just (5.8), it is probably fairly hard to demonstrate the appearance of the mass gap for gauge fluctuations.
} 
example) required to reach the continuum limit. Neither of these difficulties are present in QCD(adj) with massive fermions and deformed YM, while the problem of accessibility of the abelian confinement regime is present in all three cases. It seems to us that $\mathrm{QCD}(\mathrm{adj})$ with massive fermions and deformed YM on small $\mathbb{R}^{3} \times \mathbb{S}^{1}$ are a target of opportunity, both of which can easily be simulated. If abelian confinement regime can be reached, it may become an important playground for both lattice and analytical studies of non-perturbative physics. The abelian confinement regime has a potential to help both fields alike. If it can be achieved, it will be the first confrontation of reliable analytical methods against the reliable lattice methods.

\section{Conclusion and future work}

Our main results are:

- At sufficiently large magnetic fields, the fermion induced one-loop potential for Wilson line holonomy undergoes dimensional reduction by two-dimensions. The fermion contribution is enhanced by the density of state of the lowest Landau level.

- For massive adjoint fermions endowed with periodic boundary condition, changing magnetic field can alter the phase of the theory from a center-broken phase to a centersymmetric phase. This is an exotic phase transition induced by the competition between center-destabilizing one-loop gauge contributions and center stabilizing LLLadjoint fermion contribution.

- The fully center stabilized theory has both abelian confinement regime and nonabelian confinement regime. These two regimes are continuously connected in the sense of center symmetry, but the behavior of Wilson line eigenvalues is drastically different as shown in figure 2 .

- Realizing the abelianization (adjoint Higgsing) in lattice simulations requires the lattice version of the scaling $\frac{L_{4} N \Lambda}{2 \pi} \lesssim 1$ and $L_{i} \gtrsim \frac{L_{4} N}{2 \pi}$, and is currently feasible [42, 61]. But realizing the setting of abelian confinement regime requires an exponential hierarchy of scales, $L_{i} \gtrsim \frac{L_{4} N}{2 \pi} e^{+\frac{4 \pi^{2}}{g^{2}\left(m_{W}\right) N}}$ on a (physical) size $L_{i}^{3} \times L_{4} 4$-torus (emulating $\mathbb{R}^{3} \times \mathbb{S}^{1}$ ). This may be technically challenging, but is a worthy endeavor because of questions such as confinement and mass gap in $4 \mathrm{~d}$ non-abelian gauge theories. This is the first confrontation of reliable semi-classical methods against numerical lattice simulations.

For future work, we aim to study the deformed QCD with light fermions in the presence of large-magnetic fields, in the scaling regime $\sqrt{e B} \gg \Lambda \gg m$.

- It is already known that confinement and discrete chiral symmetry breaking can take place at weak coupling as well. Our goal is to construct a calculable theories in which both confinement and non-abelian continuous chiral symmetry breaking take place at weak coupling, and the dynamics is continuously connected to the one on $\mathbb{R}^{4}$. This may provide a useful laboratory for QCD on $\mathbb{R}^{4}$. 
- We would like to understand the role of the magnetic field on the fermonic zero and quasi-modes of monopole-instantons, and bions. These defects are exponentially more important than the $4 \mathrm{~d}$ instantons. We would like to understand how large-magnetic fields may alter index theorems for monopole-instantons, confinement mechanism, and fermion induced pairing mechanism of (chromo)-magnetic bions.

- We aim to study the effect of monopole-instantons and the sphalerons associated with monopole-instantons on the chiral magnetic effect. It is natural to expect that if $4 \mathrm{~d}$ instantons induce a chiral magnetic current or non-vanishing fluctuations, then monopole-instantons effects should enhance that by an exponential amount.

\section{Acknowledgments}

It is a pleasure to thank G. Cossu, G. Dunne, G. Basar, F. Bruckmann, D. Kharzeev, V.A. Miransky, and E. Poppitz for helpful comments. The work of M.Ü. is supported in part by DOE grant DE-FG02-12ER41806. The work of M.A. is supported by NSERC Discovery Grant of Canada.

Open Access. This article is distributed under the terms of the Creative Commons Attribution License (CC-BY 4.0), which permits any use, distribution and reproduction in any medium, provided the original author(s) and source are credited.

\section{References}

[1] D. Kharzeev, Parity violation in hot QCD: Why it can happen and how to look for it, Phys. Lett. B 633 (2006) 260 [hep-ph/0406125] [INSPIRE].

[2] D. Kharzeev and A. Zhitnitsky, Charge separation induced by P-odd bubbles in QCD matter, Nucl. Phys. A 797 (2007) 67 [arXiv:0706.1026] [InSPIRE].

[3] D.E. Kharzeev, L.D. McLerran and H.J. Warringa, The Effects of topological charge change in heavy ion collisions: 'Event by event P and CP-violation', Nucl. Phys. A 803 (2008) 227 [arXiv: 0711.0950] [INSPIRE].

[4] K. Fukushima, D.E. Kharzeev and H.J. Warringa, The Chiral Magnetic Effect, Phys. Rev. D 78 (2008) 074033 [arXiv: 0808.3382] [INSPIRE].

[5] G. Basar and G.V. Dunne, The Chiral Magnetic Effect and Axial Anomalies, Lect. Notes Phys. 871 (2013) 261 [arXiv: 1207.4199] [INSPIRE].

[6] K.G. Klimenko, Three-dimensional Gross-Neveu model in an external magnetic field, Theor. Math. Phys. 89 (1992) 1161 [INSPIRE].

[7] K.G. Klimenko, Three-dimensional Gross-Neveu model at nonzero temperature and in an external magnetic field, Theor. Math. Phys. 90 (1992) 1 [INSPIRE].

[8] V.P. Gusynin, V.A. Miransky and I.A. Shovkovy, Dimensional reduction and catalysis of dynamical symmetry breaking by a magnetic field, Nucl. Phys. B 462 (1996) 249 [hep-ph/9509320] [INSPIRE].

[9] V.P. Gusynin, V.A. Miransky and I.A. Shovkovy, Catalysis of dynamical flavor symmetry breaking by a magnetic field in (2+1)-dimensions, Phys. Rev. Lett. 73 (1994) 3499 [Erratum ibid. 76 (1996) 1005] [hep-ph/9405262] [INSPIRE]. 
[10] V.P. Gusynin, V.A. Miransky and I.A. Shovkovy, Theory of the magnetic catalysis of chiral symmetry breaking in QED, Nucl. Phys. B 563 (1999) 361 [hep-ph/9908320] [INSPIRE].

[11] G.S. Bali et al., The QCD phase diagram for external magnetic fields, JHEP 02 (2012) 044 [arXiv:1111.4956] [INSPIRE].

[12] G.S. Bali et al., QCD quark condensate in external magnetic fields, Phys. Rev. D 86 (2012) 071502 [arXiv: 1206.4205 ] [INSPIRE].

[13] G.S. Bali, F. Bruckmann, G. Endrodi, F. Gruber and A. Schaefer, Magnetic field-induced gluonic (inverse) catalysis and pressure (an)isotropy in QCD, JHEP 04 (2013) 130 [arXiv: 1303.1328] [INSPIRE].

[14] F. Bruckmann, G. Endrodi and T.G. Kovacs, Inverse magnetic catalysis and the Polyakov loop, JHEP 04 (2013) 112 [arXiv:1303.3972] [INSPIRE].

[15] M. D'Elia, S. Mukherjee and F. Sanfilippo, QCD Phase Transition in a Strong Magnetic Background, Phys. Rev. D 82 (2010) 051501 [arXiv: 1005.5365] [InSPIRE].

[16] M. D'Elia and F. Negro, Chiral Properties of Strong Interactions in a Magnetic Background, Phys. Rev. D 83 (2011) 114028 [arXiv:1103.2080] [InSPIRE].

[17] G.V. Dunne, The Heisenberg-Euler Effective Action: 75 years on, Int. J. Mod. Phys. A 27 (2012) 1260004 [arXiv: 1202.1557] [INSPIRE].

[18] G.V. Dunne, Heisenberg-Euler effective Lagrangians: Basics and extensions, hep-th/0406216 [INSPIRE].

[19] D.J. Gross, R.D. Pisarski and L.G. Yaffe, $Q C D$ and Instantons at Finite Temperature, Rev. Mod. Phys. 53 (1981) 43 [INSPIRE].

[20] G.M. Shore, Symmetry Restoration and the Background Field Method in Gauge Theories, Annals Phys. 137 (1981) 262 [inSPIRE].

[21] D. Cangemi and G.V. Dunne, Temperature expansions for magnetic systems, Annals Phys. 249 (1996) 582 [hep-th/9601048] [INSPIRE].

[22] W. Dittrich, Effective lagrangians at finite temperature, Phys. Rev. D 19 (1979) 2385 [INSPIRE].

[23] H.W. Braden, Expansions for Field Theories on $S^{\prime} \times \Sigma$, Phys. Rev. D 25 (1982) 1028 [INSPIRE].

[24] P.N. Meisinger and M.C. Ogilvie, Complete high temperature expansions for one loop finite temperature effects, Phys. Rev. D 65 (2002) 056013 [hep-ph/0108026] [INSPIRE].

[25] P.N. Meisinger and M.C. Ogilvie, The Finite temperature SU(2) Savvidy model with a nontrivial Polyakov loop, Phys. Rev. D 66 (2002) 105006 [hep-ph/0206181] [INSPIRE].

[26] M. Ünsal and L.G. Yaffe, (In)validity of large- $N$ orientifold equivalence, Phys. Rev. D 74 (2006) 105019 [hep-th/0608180] [INSPIRE].

[27] A.J. Mizher, M.N. Chernodub and E.S. Fraga, Phase diagram of hot QCD in an external magnetic field: possible splitting of deconfinement and chiral transitions, Phys. Rev. D 82 (2010) 105016 [arXiv: 1004.2712] [INSPIRE].

[28] A. Armoni, M. Shifman and G. Veneziano, Exact results in nonsupersymmetric large- $N$ orientifold field theories, Nucl. Phys. B 667 (2003) 170 [hep-th/0302163] [INSPIRE].

[29] A. Armoni, M. Shifman and G. Veneziano, SUSY relics in one flavor QCD from a new $1 / N$ expansion, Phys. Rev. Lett. 91 (2003) 191601 [hep-th/0307097] [INSPIRE]. 
[30] Y. Hosotani, Dynamics of Nonintegrable Phases and Gauge Symmetry Breaking, Annals Phys. 190 (1989) 233 [inSPIRE].

[31] A. Higuchi and L. Parker, Aspects of QED and Nonabelian Gauge Theories in $S_{1} \times R_{3}$ and $S_{1} \times R_{4}$ Space-times, Phys. Rev. D 37 (1988) 2853 [InSPIRE].

[32] A.T. Davies and A. McLachlan, Gauge group breaking by Wilson loops, Phys. Lett. B 200 (1988) 305 [INSPIRE].

[33] M. Ünsal, Magnetic bion condensation: A New mechanism of confinement and mass gap in four dimensions, Phys. Rev. D 80 (2009) 065001 [arXiv:0709.3269] [INSPIRE].

[34] P. Kovtun, M. Ünsal and L.G. Yaffe, Volume independence in large- $N_{c}$ QCD-like gauge theories, JHEP 06 (2007) 019 [hep-th/0702021] [INSPIRE].

[35] T. Eguchi and H. Kawai, Reduction of Dynamical Degrees of Freedom in the Large- $N$ Gauge Theory, Phys. Rev. Lett. 48 (1982) 1063 [inSPIRE].

[36] M. Ünsal and L.G. Yaffe, Center-stabilized Yang-Mills theory: Confinement and large- $N$ volume independence, Phys. Rev. D 78 (2008) 065035 [arXiv:0803.0344] [INSPIRE].

[37] M. Ünsal and L.G. Yaffe, Large- $N$ volume independence in conformal and confining gauge theories, JHEP 08 (2010) 030 [arXiv:1006.2101] [INSPIRE].

[38] A. Armoni, D. Dorigoni and G. Veneziano, k-String Tension from Eguchi-Kawai Reduction, JHEP 10 (2011) 086 [arXiv:1108.6196] [InSPIRE].

[39] M. Garcia Pérez, A. González-Arroyo and M. Okawa, Spatial volume dependence for $2+1$ dimensional $\mathrm{SU}(N)$ Yang-Mills theory, JHEP 09 (2013) 003 [arXiv:1307.5254] [INSPIRE].

[40] B. Lucini and M. Panero, SU(N) gauge theories at large-N, Phys. Rept. 526 (2013) 93 [arXiv: 1210.4997] [INSPIRE].

[41] B. Lucini and M. Panero, Introductory lectures to large-N QCD phenomenology and lattice results, Prog. Part. Nucl. Phys. 75 (2014) 1 [arXiv:1309.3638] [InSPIRE].

[42] G. Cossu and M. D'Elia, Finite size phase transitions in QCD with adjoint fermions, JHEP 07 (2009) 048 [arXiv: 0904.1353] [INSPIRE].

[43] Y. Hosotani, Dynamical Mass Generation by Compact Extra Dimensions, Phys. Lett. B 126 (1983) 309 [INSPIRE].

[44] P.C. Argyres and M. Ünsal, The semi-classical expansion and resurgence in gauge theories: new perturbative, instanton, bion and renormalon effects, JHEP 08 (2012) 063 [arXiv: 1206.1890] [INSPIRE].

[45] K. Kashiwa and T. Misumi, Phase structure and Hosotani mechanism in gauge theories with compact dimensions revisited, JHEP 05 (2013) 042 [arXiv:1302.2196] [INSPIRE].

[46] H. Kouno et al., Differences and similarities between fundamental and adjoint matters in SU(N) gauge theories, Phys. Rev. D 88 (2013) 016002 [arXiv: 1304.3274] [InSPIRE].

[47] C. Bonati, M. D'Elia, M. Mariti, F. Negro and F. Sanfilippo, Magnetic Susceptibility of Strongly Interacting Matter across the Deconfinement Transition, Phys. Rev. Lett. 111 (2013) 182001 [arXiv:1307.8063] [INSPIRE].

[48] M.C. Ogilvie, Phases of Gauge Theories, J. Phys. A 45 (2012) 483001 [arXiv:1211.2843] [INSPIRE].

[49] P.N. Meisinger and M.C. Ogilvie, String Tension Scaling in High-Temperature Confined $\mathrm{SU}(N)$ Gauge Theories, Phys. Rev. D 81 (2010) 025012 [arXiv:0905.3577] [InSPIRE]. 
[50] E. Thomas and A.R. Zhitnitsky, Topological Susceptibility and Contact Term in QCD. A Toy Model, Phys. Rev. D 85 (2012) 044039 [arXiv: 1109.2608] [inSPIRE].

[51] A.R. Zhitnitsky, P odd fluctuations and Long Range Order in Heavy Ion Collisions. Deformed QCD as a Toy Model, Nucl. Phys. A 897 (2013) 93 [arXiv:1208.2697] [InSPIRE].

[52] A.R. Zhitnitsky, QCD as a topologically ordered system, Annals Phys. 336 (2013) 462 [arXiv: 1301.7072] [INSPIRE].

[53] H. Vairinhos, Monte Carlo Algorithms For Reduced Lattices, Mixed Actions, And Double-Trace Deformations, arXiv:1010.1253 [INSPIRE].

[54] H. Vairinhos, Phase transitions in center-stabilized lattice gauge theories, PoS (Lattice 2011) 252 [arXiv: 1111.0303] [INSPIRE].

[55] M. Shifman and M. Ünsal, QCD-like Theories on $R(3) \times S(1)$ : A Smooth Journey from Small to Large r(S(1)) with Double-Trace Deformations, Phys. Rev. D 78 (2008) 065004 [arXiv: 0802.1232] [inSPIRE].

[56] G. Endrödi, QCD equation of state at nonzero magnetic fields in the Hadron Resonance Gas model, JHEP 04 (2013) 023 [arXiv: 1301.1307] [INSPIRE].

[57] V.A. Miransky and I.A. Shovkovy, Magnetic catalysis and anisotropic confinement in QCD, Phys. Rev. D 66 (2002) 045006 [hep-ph/0205348] [INSPIRE].

[58] E. Poppitz, T. Schäfer and M. Ünsal, Universal mechanism of (semi-classical) deconfinement and theta-dependence for all simple groups, JHEP 03 (2013) 087 [arXiv:1212.1238] [INSPIRE].

[59] N. Seiberg and E. Witten, Gauge dynamics and compactification to three-dimensions, hep-th/9607163 [INSPIRE].

[60] A.M. Polyakov, Quark Confinement and Topology of Gauge Groups, Nucl. Phys. B 120 (1977) 429 [INSPIRE].

[61] G. Cossu, H. Hatanaka, Y. Hosotani and J. Noaki, Polyakov loops and the Hosotani mechanism on the lattice, to appear. 